\title{
A escolha do parto e a participação do enfermeiro obstetra: visão de acadêmicos de enfermagem
}

\author{
Isabela dos Santos Benites*, Giselle Juliana de Jesus*, Zaida Aurora Sperli Soler, D.Sc.**
}

\begin{abstract}
*Acadêmica de enfermagem, Faculdade de Medicina de Sáo José do Rio Preto-SP FAMERP - 40 ano de Enfermagem, ${ }^{* *}$ Docente da Graduação e da Pós-Graduação da Faculdade de Medicina de São José do Rio Preto (FAMERP), Orientadora em nível de Mestrado e Doutorado da FAMERP, Diretora Adjunta de Extensáo de Serviços à Comunidade da FAMERP
\end{abstract}

\begin{abstract}
Resumo
Introdução: Os índices absurdos de cesarianas no Brasil têm sido discutidos no meio científico mundial, e muitas intervençôes são recomendadas, como a formação em nível de especialização do enfermeiro obstetra, para atuação efetiva na atenção à mulher no ciclo gravídico puerperal, no enfoque da humanização do nascimento e alcance de maiores índices de parto normal-natural, durante o ciclo gravídico-puerperal, particularmente no trabalho de parto e o parto. Objetivo: Identificar entre acadêmicos de enfermagem a percepção que têm sobre o nascimento, a escolha do tipo de parto e a atuação do enfermeiro obstetra na assistência. Métodos: O projeto deste estudo foi aprovado por Comitê de Ética em Pesquisa (CEP). Consentiram em participar da pesquisa 142 graduandas do primeiro ao quarto ano de um curso de graduação em enfermagem de uma autarquia estadual. Para a obtenção dos dados foi usado um questionário com questóes semiestruturadas e abertas, abordando as temáticas da escolha do tipo de parto e sobre a atuação do enfermeiro obstetra. Resultados: Os principais resultados foram: $66,9 \%$ escolheria o parto normal; durante a graduaçáo $50 \%$ das acadêmicas tinham visto parto normal e $74,4 \%$ já tinham presenciado cesariana; $80 \%$ referiram que gostariam de ter seu trabalho de parto e o parto acompanhado por enfermeiro obstetra. Conclusão: Os resultados deste estudo revelaram que as acadêmicas de enfermagem, principalmente da terceira e quarta séries, têm mais conhecimento sobre os benefícios do parto normal e sobre as especificidades de formação e atuação do enfermeiro obstetra.
\end{abstract}

Palavras-chave: parto normal, cesárea, enfermagem obstétrica, parto humanizado, saúde da mulher.

\section{Abstract}

\section{Childbirth choice and the obstetric nurse participation: nursing academics vision}

Introduction: The highest rates of cesarean sections in Brazil have been discussed in the scientific world and many interventions are recommended, such as the training level of expertise of the obstetric nurse, to work effectively in the care of women during pregnancy and pueperium, approaching the humanization of birth and achievement of higher rates of normal-natural childbirth. Objective: To identify among nursing students their perception about the birth; the choice of type of childbirth and the role of an obstetric nurse on this care during the pregnancy and childbirth, particularly in labor 
and childbirth. Method: The study design was approved by the Ethics Committee in Research (CEP). A hundred forty two students from the first to fourth grades of a Nursing graduation course-state autarchy consented in participating. A questionnaire with semi-structured and open questions addressing the issues of choice of childbirth type and the role of the obstetric nurse was used. Results: The main results were: $66.9 \%$ would choose normal childbirth; during graduation $50 \%$ of undergraduate students had seen normal childbirth and $74.4 \%$ had seen cesarean, $80 \%$ said they would like to have their labor and childbirth followed by an obstetric nurse. Conclusion: The results of this study showed that the nursing students, mainly from the third and fourth grades have more knowledge about the benefits of natural childbirth and on the specifics of training and performance of the obstetric nurse.

Key-words: normal childbirth, cesarean section, obstetrics nursing, humanized labor, women's health.

\section{Resumen}

\section{La elección del tipo de parto y la participación del enfermero obstetra: la visión de académicos de enfermería}

Introducción: Los índices excesivos de cesáreas en Brasil se discuten en el medio científico mundial. Se recomiendan intervenciones, como la formación a nivel de Especialización del enfermero obstetra, para la actuación efectiva en la atención a la mujer en el ciclo gravídico-puerperal en el enfoque de la humanización del nacimiento y para alcanzar mayores índices de parto normal-natural durante el ciclo gravídico puerperal, especialmente en el trabajo de parto y en el parto. Objetivo: Identificar la percepción de académicos de enfermería sobre el nacimiento, la elección del tipo de parto y la actuación del enfermero obstetra en la asistencia. Método: El Proyecto de estudio fue analizado y aprobado por el Comité de Ética en Investigaciones (CEI). 142 estudiantes del primero al cuarto año de un curso de graduación en Enfermería de una autarquía estadual aceptaron participar del estudio. Para la obtención de los datos, se utilizó un cuestionario con preguntas semi-estructuradas y abiertas, que abarcaban temas como la elección del tipo de parto y la actuación del enfermero obstetra. Resultados: Los principales resultados fueron: el 66,9\% elegiría el parto normal; durante la graduación, el $50 \%$ de las académicas había visto un parto normal y el $74,4 \%$ ya había visto una cesárea; el $80 \%$ relató que les gustaría tener la asistencia de un enfermero obstetra durante su trabajo de parto y el parto. Conclusión: Los resultados de este estudio revelaron que las académicas de enfermería, principalmente del tercero y cuarto año de graduación, poseen un mayor conocimiento sobre las ventajas del parto normal y sobre las especificidades de formación y actuación del enfermero obstetra.

Palabras-clave: parto normal, cesárea, enfermería obstétrica, parto humanizado, salud de la mujer.

\section{Introdução}

A gestação pode ser compreendida como um momento especial na vida da mulher. Pode-se considerar que, em meio a tantos eventos ocorridos nesta fase, o ato do parto é, sem dúvida, o mais temido pelas mulheres [1]. A escolha pelo tipo de parto é um assunto que há décadas vem sendo muito discutido. Fatores como a escolaridade, nível socioeconômico, experiências anteriores e o acesso à informação pela mídia e internet influenciam a decisão da mulher quanto à via de resolução do parto [2-3].

Durante muito tempo, o trabalho de parto foi considerado uma prática feminina sendo realizado por mulheres experientes denominadas parteiras no seio da estrutura familiar [4]. No entanto, aos poucos o cenário do nascimento foi se modificando, com o avanço da tecnologia, do progresso científico relacionado aos procedimentos cirúrgicos e à utili- zação de fármacos, levando ao aumento da hospitalização, passando a realizaçáo do parto da esfera domiciliar para instituições de saúde, distanciando-se cada vez mais de seu processo natural [5].

No Brasil fica evidente a ocorrência de distorçôes na assistência obstétrica a partir da década de 70, com índices abusivos de cesárea e a excessiva medicalização durante o ciclo gravídico puerperal, deixando a mulher submissa a regras ditadas pelos profissionais e instituiçôes de saúde [4].

Vários aspectos também são ressaltados sobre a evolução da participação de profissionais na assistência ao nascimento culminando na mudança da via de parto vaginal/ natural para cirúrgica/ cesárea [4]. A conduta do profissional médico também pode influenciar a decisão da mulher principalmente nos momentos finais da gestação [6]. Estes, por sua vez, alegam que a realização da cesariana muitas vezes é um desejo da gestante justificado pelo medo da 
dor durante o trabalho de parto e a ocasiáo que possibilita a realização da laqueadura tubária [7].

Cabe ressaltar que o uso desordenado da cesárea aumenta as chances de complicaçóes durante o trabalho de parto sendo, portanto, indicada somente nos casos em que o parto normal colocará em risco a vida da mãe e do feto, pois sendo um procedimento invasivo oferece maior risco de infecção, possibilidade de laceração de outros órgáos e problemas relacionados à cicatrização. $\mathrm{O}$ parto normal sendo um processo natural oferece diversos benefícios e é o mais indicado [4-8,9].

O Ministério da Saúde (MS) ressalta que o parto normal deve ser estimulado, respeitando a fisiologia natural do parto, resgatando e valorizando a autonomia da mulher [10]. A Organização Mundial da Saúde (OMS) [11] aponta que 70 a $80 \%$ das gestaçóes podem ser consideradas de baixo risco e recomenda que a taxa de cesarianas náo exceda a $15 \%$. Afirma também que o Enfermeiro Obstetra apresenta o perfil mais indicado a prestar assistência ao parto normal [11]. Entretanto, uma pesquisa realizada com profissionais médicos revelou que muitos desconhecem a legislação que ampara o enfermeiro obstetra e revela também que, em sua maioria, não sabem definir o papel deste profissional [12]

No Brasil, a Lei $n^{\circ} 7.498 / 86$, regulamentada pelo Decreto 94.406/87 que dispóe sobre o exercício da enfermagem e dá outras providências, descreve em seu artigo $11^{\circ}$ que além das atividades e competências do profissional enfermeiro, cabe ao titular do diploma ou certificado de Obstetriz ou de Enfermeira Obstétrica, a incumbência de: assistir à parturiente e ao parto normal; identificar distócias obstétricas e tomar providências até a chegada do médico; realizar episiotomia e episiorrafia e aplicar anestesia local, quando necessário [13]

Embora muitos estudos pontuem o enfermeiro obstetra como um profissional preparado e qualificado, ainda é necessário maior reconhecimento de suas competências e valorização de sua atuação na assistência a mulher e ao recém-nascido durante o ciclo gravídico-puerperal [14].

Contudo, diante de questionamentos e debates que norteiam a escolha pela via de parto e o tipo de profissional envolvido, é importante conhecermos a opiniâo daquela que poderá atuar direta ou indiretamente na assistência ao parto de outras mulheres - a acadêmica de enfermagem.

Portanto, este estudo realizado entre acadêmicas de enfermagem da FAMERP, teve como objetivos:
1. Caracterizar as acadêmicas quanto a variáveis sociais;

2. Verificar qual o tipo de parto de escolha, suas justificativas e experiências, bem como explorar seus conhecimentos em relação ao papel do enfermeiro obstetra na atenção ao nascimento.

\section{Material e métodos}

Trata-se de um estudo quantitativo, exploratório e descritivo quanto à escolha do parto e papel da enfermeira obstetra, às perspectivas e aos fatores determinantes na visão de acadêmicos de enfermagem. O estudo foi realizado na Faculdade de Medicina de Sáo José do Rio Preto (FAMERP), uma instituiçáo de ensino pública, autarquia estadual, mantida pelo Governo do Estado de São Paulo e que oferece os cursos de graduação em Medicina e Enfermagem.

A população do estudo foi composta por 142 acadêmicas de enfermagem da FAMERP, do sexo feminino, regularmente matriculadas no período de 2008 a 2011, da $1^{\mathrm{a}}$ a $4^{\mathrm{a}}$ série no ano de 2011. Foram excluídos os casos em que houve trancamento de matrícula.

O instrumento de coleta de dados (Anexo 1) aplicado contemplou 10 questóes semiabertas que visavam identificar a preferência das acadêmicas de enfermagem pelo parto normal ou cesariana bem como suas justificativas e perspectivas quanto ao papel da enfermeira obstetra. Também contemplou questóes para que as participantes que já vivenciaram a experiência de parir pudessem expor suas experiências.

O estudo foi submetido e aprovado pelo Comitê de Ética em Pesquisa (CEP) segundo protocolo $n^{\circ} 3921 / 2011$. Após esclarecimento do estudo e apresentação dos responsáveis, os participantes consentiram responder o questionário respeitando-se as Diretrizes e Normas Regulamentadoras de Pesquisas em Seres Humanos.

Para atender os objetivos propostos no estudo, variáveis de caracterização amostral como série da graduação, sexo e estado civil e variáveis avaliaram a preferência pela via de parto e as perspectivas quanto ao parto normal realizado pelo enfermeiro obstetra.

Os resultados do estudo foram expressos por meio de tabelas e valores percentuais, além de testes estatísticos de associaçáo que foram aplicados por meio da avaliação da estatística qui-quadrado, adotando nível de significância de 0,05. 


\section{Resultados}

Os resultados do estudo baseiam-se na análise das respostas de 142 graduandas de enfermagem. Os resultados da Tabela I mostram que: todos os respondentes eram do sexo feminino (142-100\%); solteiras (140-98,6\%); estudantes da $1^{\text {a }}$ série do curso de Enfermagem (43-30,3\%). A maioria dos respondentes escolheria o parto normal como via de parto (95-66,9\%), por ser mais saudável, menos invasivo e por ter melhor recuperaçáo $(82-71,3 \%)$. A maioria nasceu por cesariana $(112-78,9 \%)$, já presenciou algum tipo de parto (79-55,6\%), já assistiu parto normal em estágios na graduação (30$50,0 \%)$, já presenciou parto cesariana em estágios na graduação $(58-74,4 \%)$ e gostaria que o parto fosse realizado por um enfermeiro (112-80\%). A maioria dos respondentes mostrou que o papel do enfermeiro obstetra está vinculado a acompanhar a gestação e realizar o parto normal (44-35,2\%).

Cabe ressaltar que a idade mínima verificada foi de 17 anos e a máxima de 37 anos. A idade dos respondentes apresentou média de 20,95 anos com desvio padrão de 2,36 anos e mediana de 20,00 anos. Foi observada presença de valores discrepantes (outliers) - sendo eles as idades referentes a 28 e 37 anos - que influenciaram no valor da média da idade.

Tabela I - Percentuais referentes às variáveis de caracterização amostral das de opinião das acadêmicas de enfermagem do estudo. São José do Rio Preto, 2011.

\begin{tabular}{lcc}
\hline \multicolumn{1}{c}{ Variáveis de caracterização } & $\mathbf{n}$ & $\%$ \\
\hline Sexo & 142 & 100 \\
\hline Masculino & 0 & 0,00 \\
Feminino & 142 & 100 \\
Estado civil & 142 & 100 \\
Divorciada & 2 & 1,41 \\
Solteira & 140 & 98,59 \\
\hline Série da graduação & 142 & 100 \\
\hline $1^{\text {a }}$ & 43 & 30,28 \\
$2^{a}$ & 41 & 28,87 \\
$3^{a}$ & 40 & 28,17 \\
$4^{\text {a }}$ & 18 & 12,68 \\
\hline Escolha da via do parto & 142 & 100 \\
\hline Cesariana & 47 & 33,10 \\
Normal & 95 & 66,90 \\
\hline Motivo da escolha & 115 & 100 \\
\hline Escolha da data e medo da dor e & 33 & 28,70 \\
de complicações (cesariana) & & \\
Mais saudável, menos invasivo, & 82 & 71,30 \\
melhor recuperação (normal) & & \\
\hline
\end{tabular}

\begin{tabular}{lcc}
\hline Via do parto no nascimento & 142 & 100 \\
\hline Cesariana & 112 & 78,87 \\
Normal & 30 & 21,13 \\
\hline Presenciou ou assistiu algum parto & 142 & 100 \\
\hline Não & 63 & 44,37 \\
Sim & 79 & 55,63 \\
\hline Presenciou ou assistiu parto & 60 & 100 \\
normal & 30 & 50,00 \\
\hline Estágio na graduação & 4 & 6,67 \\
$\begin{array}{l}\text { Outros } \\
\text { Vídeos na internet }\end{array}$ & 26 & 43,33 \\
\hline $\begin{array}{l}\text { Presenciou ou assistiu parto cesa- } \\
\text { riana }\end{array}$ & 78 & 100 \\
\hline Estágio na graduação & 58 & 74,36 \\
$\begin{array}{l}\text { Outros } \\
\text { Vídeos na internet }\end{array}$ & 12 & 15,38 \\
\hline Gostaria que o parto fosse realiza- & 140 & 10,26 \\
\hline do pelo enfermeiro & 100 \\
\hline Não & 28 & 20,00 \\
Sim & 112 & 80,00 \\
\hline $\begin{array}{l}\text { Qual o papel do enfermeiro } \\
\text { obstetra }\end{array}$ & 125 & 100 \\
\hline Acompanha a gestação e faz o \\
$\begin{array}{l}\text { parto normal } \\
\text { Auxilia no parto normal }\end{array}$ & 44 & 35,20 \\
\hline Não tem conhecimento do papel \\
do enfermeiro & 14 & 11,20 \\
\hline $\begin{array}{l}\text { Orienta a gestante } \\
\text { Pode realizar o parto normal }\end{array}$ & 24 & 26,40 \\
\hline & 10,00 \\
\hline
\end{tabular}

As respostas das acadêmicas do estudo quanto à escolha da via de parto, segundo a série de graduação. Verifica-se que há um predomínio da escolha pelo parto normal, porém essa escolha torna-se mais evidente na $3^{\mathrm{a}}$ e $4^{\mathrm{a}}$ série, visto que existe um percentual considerável de alunas do $1^{\circ}$ e $2^{\circ}$ ano da graduação que optaram cesariana como via de parto (Tabela II).

Tabela II - Distribuição percentual da escolha da via do parto pelas acadêmicas de Enfermagem do estudo, segundo a série da graduação. São José do Rio Preto, 2011.

\begin{tabular}{lccc}
\hline \multirow{2}{*}{ Série } & \multicolumn{2}{c}{ Escolha da via do parto } & Total \\
\cline { 2 - 4 } & Cesariana & Normal \\
\hline $1^{\text {a }}$ & $19(44,19 \%)$ & $24(55,81 \%)$ & $43(30,28 \%)$ \\
$2^{a}$ & $18(43,90 \%)$ & $23(56,10 \%)$ & $41(28,87 \%)$ \\
$3^{a}$ & $8(20,00 \%)$ & $32(80,00 \%)$ & $40(28,17 \%)$ \\
$4^{\text {a }}$ & $2(11,11 \%)$ & $16(88,89 \%)$ & $18(12,68 \%)$ \\
Total & $47(33,10 \%)$ & $95(66,90 \%)$ & $142(100 \%)$ \\
Valor P & \multicolumn{3}{c}{0,006} \\
\hline
\end{tabular}


$\mathrm{Na}$ Tabela III mostra-se a opiniáo das acadêmicas quanto à condução do parto pelo enfermeiro obstetra e ser assistida por ele no parto normal. Verifica-se que a opção por este profissional é maior na $3^{\mathrm{a}}(36-90 \%)$ e na $4^{\mathrm{a}}(17-94,4 \%)$ séries. Os alunos das séries iniciais náo anotaram que escolheriam o enfermeiro na condução e realizaçáo do parto normal.

Os resultados mostram que a maioria dos alunos da $1^{\text {a }}$ série relatou que o enfermeiro obstetra auxilia no parto normal (8-24,24\%), a maioria dos alunos da $2^{\mathrm{a}}$ série relatou que não tem conhecimento sobre a função do enfermeiro obstetra (18-47,37\%), sendo que a maioria dos alunos da $3 \mathrm{a}(30-75,00 \%)$ e da 4a (9-64,29\%) séries relatou que a função do enfermeiro obstetra é acompanhar a gestação e fazer o parto.

\section{Discussão}

A decisão pelo tipo de parto está associada a diversos fatores. A preferência pelo tipo de parto das acadêmicas de enfermagem referidas neste estudo mostrou-se estar associada diretamente ao nível de informação.

Em todos os anos de graduaçáo predominou a escolha pelo parto normal, entretanto, a maioria esteve associada às alunas que tiveram maior conhecimento na graduação durante disciplina relacionada à temática bem como oportunidade de presenciar o parto normal em "locu".

Cabe aqui informar que, aos acadêmicos da $3^{\mathrm{a}}$ e $4^{\mathrm{a}}$ série de enfermagem da FAMERP é oferecida no terceiro ano da graduaçáo a disciplina de Saúde da Mulher, quando têm oportunidade de receber informaçóes importantes durante a assistência de enfermagem prestada à mulher nas diferentes etapas do ciclo de vida, principalmente durante o ciclo gravídico- puerperal.

Com base na informação anterior e resultados obtidos conclui-se que, à medida que o aluno passa da segunda para a terceira série bem como pela disciplina de saúde da mulher ocorre uma mudança na escolha pela via de parto. Na disciplina de Saúde da Mulher os alunos desenvolvem o conhecimento teórico durante estágio no Hospital de Base-HB, Ambulatório de Especialidades e em Unidade básica de Saúde de São José do Rio Preto. No campo hospitalar os alunos têm a oportunidade de acompanhar a assistência oferecida à mulher desde o trabalho de parto, o parto em si (cesárea e normal) e o período pós-parto. Com base nessas informaçóes, os alunos também foram questionados sobre a possibilidade de já terem visualizado o parto normal e a forma que lhes possibilitou essa visualização.

Num estudo realizado com mulheres que optaram pelo parto normal domiciliar revelou que estas apenas decidiram após sentir-se plenamente informadas sobre os diferentes modelos de assistência ao parto [15].

O nível de informação das mulheres está fortemente ligado à condição socioeconômica e nível de escolaridade, entretanto, no Brasil a maioria das mulheres não tem acesso aos conhecimentos necessários e adequados. Uma pesquisa realizada com dez gestantes que analisou o nível de informação e influências para a decisão do tipo de parto revelou que a família e a mídia são agentes importantes na decisão do tipo de parto, sendo esta última, geralmente influenciadora na escolha pela cesárea associada ao elevado número de notícias sobre partos complicados e a demora das gestantes que tiveram parto normal para serem atendidas. Ainda, a escolha pela via de parto cesariana esteve direcionada

Tabela III - Percentuais do conhecimento das acadêmicas de enfermagem do estudo quanto ao papel do enfermeiro obstetra na condução do parto, segundo a série da graduação. São José do Rio Preto, 2011.

\begin{tabular}{lccccc}
\hline \multicolumn{1}{c}{$\begin{array}{c}\text { Papel do enfermeiro } \\
\text { obstetra }\end{array}$} & $1^{\mathbf{a}}$ & $2^{\text {a }}$ & $3^{\mathbf{a}}$ & $4^{\text {a }}$ & Tórie \\
\cline { 2 - 5 } & $2(6,06 \%)$ & $3(7,89 \%)$ & $30(75,00 \%)$ & $9(64,29 \%)$ & $44(35,20 \%)$ \\
Acompanha a gestação e & $8(24,24 \%)$ & $5(13,16 \%)$ & $1(2,50 \%)$ & $0(0,00 \%)$ & $14(11,20 \%)$ \\
faz o parto & $15(45,45 \%)$ & $18(47,37 \%)$ & $0(0,00 \%)$ & $0(0,00 \%)$ & $33(26,40 \%)$ \\
Auxilia no parto normal & $1(3,03 \%)$ & $2(5,26 \%)$ & $3(7,50 \%)$ & $4(28,57 \%)$ & $10(8,00 \%)$ \\
Não tem conhecimento & $7(21,21 \%)$ & $10(26,32 \%)$ & $6(15,00 \%)$ & $1(7,14 \%)$ & $14(19,20 \%)$ \\
Orienta a gestante & $33(26,40 \%)$ & $38(30,40 \%)$ & $40(32,00 \%)$ & $14(11,20 \%)$ & $140(100 \%)$ \\
Pode realizar o parto & & & $<0,001$ & & \\
normal & & &
\end{tabular}


as alunas dos anos iniciais da graduaçáo que náo tiveram detalhadamente as informações quanto ao mecanismo do parto e risco-benefícios, posiçóes de parto relatado e que visualizaram o parto normal apenas por vídeos na internet [16].

Quanto à forma de visualização do parto normal, verificou- se que das 56 alunas que referiram ter visualizado este tipo de parto, (12-21,43\%) são da $1^{\mathrm{a}}$ e $2^{\mathrm{a}}$ séries e só tiveram a oportunidade de assisti-lo por meio de vídeos na internet, ao passo que metade das alunas da $3^{\mathrm{a}}$ série (14-50,00\%) e todas as acadêmicas da $4^{\mathrm{a}}$ série (16-100\%) o assistiram em estágios durante a graduação.

Em relação à justificativa pela escolha da via de parto, foi possível observar que as alunas que escolheram a cesariana relataram como motivos principais a possibilidade de escolher a da data do parto, medo da dor e/ou de complicaçóes durante o parto normal. A escolha do parto normal esteve vinculada ao fato de ser mais saudável, menos invasivo e promover melhor recuperação para a mulher após o nascimento.

A escolha pelo parto normal demonstrou-se fortemente ligada as acadêmicas que tiveram maior conhecimento quanto aos tipos de parto e oportunidade de presenciar a realidade de mulheres que tiveram esta vivência, podendo certamente, quebrar muitos tabus impostos pela sociedade e também pela mídia. Com isso, percebe-se que o papel do profissional no atendimento a gestante bem como as informaçóes fornecidas podem ser determinantes no processo de decisáo da mulher.

Cabe ressaltar que, embora a autonomia da mulher deva ser estimulada, é necessário compreender que nem sempre a mulher deseja tomar a decisão sozinha [17]. No entanto, cabe aos profissionais durante esta participaçáo a responsabilidade de fornecer informaçóes adequadas à mulher que possam aliviar seus medos e anseios.

Nesta pesquisa também se explorou o conhecimento das acadêmicas de enfermagem quanto ao papel do enfermeiro obstetra e a preferência pela realização do parto por este tipo de profissional. Verificou-se que grande parte dos alunos da $1^{a}$ série relatou que cabe ao enfermeiro obstetra auxiliar no parto normal, a maioria dos alunos da $2^{\mathrm{a}}$ série referiu não ter conhecimento sobre a funçáo do enfermeiro obstetra $(18-47,37 \%)$, já a maioria dos alunos da $3^{\mathrm{a}}(30-75,00 \%)$ e da $4^{\mathrm{a}}(9-64,29 \%)$ séries relatou que a função do enfermeiro obstetra é oferecer assistência durante e após a gestação e que este pode realizar o parto normal.
O enfermeiro obstetra apresenta-se como um profissional capacitado para atuar na assistência durante o ciclo gravídico puerperal. A maioria dos alunos que referiram conhecer a função do enfermeiro obstetra foram os que tiveram a oportunidade de conhecer melhor sobre o profissional na graduação - os alunos da $3^{\mathrm{a}}$ e 4 série. Uma considerável parte dos respondentes das séries iniciais não soube definir corretamente.

\section{Conclusão}

As acadêmicas do estudo recebem orientação a partir da $3^{\mathrm{a}}$. série sobre os diferentes aspectos relacionados ao nascimento, conduçáo do parto e realizaçáo do parto normal por um enfermeiro obstetra. Isso lhes permite uma opção mais fundamentada quanto à escolha da via do parto e da legalidade da assistência prestada pelo enfermeiro obstetra.

Embora o enfermeiro obstetra seja capacitado bem como indicado na assistência a mulheres durante o ciclo gravídico puerperal, é necessário o desenvolvimento de programas que valorizem e ressaltem as competências deste profissional considerando- o como um ponto estratégico na mudança do modelo atual do nascimento.

\section{Referências}

1. Tedesco RP, Maia Filho NL, Mathias L, Benez AL, Castro VCL, Bourroul GM et al. Fatores determinantes para as expectativas de primigestas acerca da via de parto. Rev Bras Ginecol Obstet 2004;26(10):791-8.

2. Mandarino NR, Chein MBC,Monteiro Júnior FC, Brito LMO, Lamy ZC, Nina VJS, et al. Aspectos relacionados à escolha do tipo de parto: um estudo comparativo entre uma maternidade pública e outra privada em São Luís, Maranhão, Brasil. Cad Saúde Pública 2009;25(7):1587-96.

3. Schreiner M, Costa JSD, Olinto MTA, Meneghel SN. Assistência ao parto em São Leopoldo (RS): um estudo de base populacional. Ciênc Saúde Coletiva 2010;15(Suppl 1):1411-6.

4. Soler ZASG. CEHPAN - Centro Humanizado de Preparo e Assistência ao Nascimento uma proposta para a região de São José do Rio Preto, São Paulo [tese]. São José do Rio Preto: Faculdade de Medicina de São José do Rio Preto; 2005.

5. Gonçalves R, Aguiar CA, Merighi MAB, Jesus MCP. Vivenciando o cuidado no contexto de uma casa de parto: o olhar das usuárias. Rev Esc Enferm USP 2011;45(1):62-70.

6. Queiroz MVO, Silva NSJ, Jorge MSB, Moreira TMM. Incidência e características de cesáreas e de partos normais: estudo em uma cidade no interior do Ceará. Rev Bras Enferm 2005;58(6):687-91. 
7. Freitas PF, Drachler ML, Leite JCC, Grassi PR. Desigualdade social nas taxas de cesariana em primíparas no Rio Grande do Sul. Rev Saúde Pública 2005;39(5):761-7.

8. Machado Junior LC, Sevrin CE, Oliveira E, Carvalho HB, Zamboni JW, Araújo JC, et al. Associação entre a via de parto e complicaçóes maternas em hospital público da Grande São Paulo, Brasil. Cad Saúde Pública 2009;25(1):124-32.

9. Sakae TM, Freitas PF, D’Orsi E. Fatores associados a taxas de cesárea em hospital universitário. Rev Saúde Pública 2009;43(3):472-80.

10. Ministério da Saúde. Secretaria de Políticas de Saúde. Área Técnica de Saúde da Mulher. Parto, aborto e puerpério. Assistência humanizada à mulher. Brasília: Ministério da Saúde; 2001.

11. Organização Mundial da Saúde (OMS). Assistência ao parto normal: um guia prático. Genebra: OMS; 1996.

12. Araújo NRA, Oliveira SC. A visão do profissional médico sobre a atuaçáo da enfermeira obstetra no centro obstétrico de um Hospital Escola de cidade do Recife
- PE. Cogitare Enferm 2006;11(1):31-8.

13. Conselho Federal de Enfermagem. Legislação. Lei $\mathrm{n}^{\circ} 7.498 / 86$ de 25 de junho de 1986. Dispóe sobre o exercício da enfermagem e dá outras providências. Brasília: Diário Oficial da União; 1986.

14. Rabelo LR, Oliveira, DL. Percepçóes de enfermeiras obstétricas sobre sua competência na atenção ao parto normal hospitalar. Rev Esc Enferm USP 2010;44(1):213-20.

15. Medeiros RMK, Santos IMM, Silva LR. A escolha pelo parto domiciliar: história de vida de mulheres que vivenciaram esta experiência. Esc Anna Nery Rev Enferm 2008;12(4):765-72.

16. Silva HM, Ribeiro CD, Costa AR. Acompanhamento de gestantes: nível de informação e influências de familiares, amigos e da mídia para a decisão do tipo de parto. Conexão Ciênc 2011;6(1):32-40.

17. Sodré TM, Merighi MAB, Bonadio IC. Escolha informada no parto: um pensar para o cuidado centrado nas necessidades da mulher. Ciênc Cuid Saúde 2012;11(Suppl):115-20.

\section{Anexo1}

Segue abaixo o parecer e instrumento utilizado para coleta de dados.

Instrumento de coleta de dados

Identificação

Nome:

Idade:

Estado Civil: ( ) A- Solteira ( )B-Casada

( )C-Divorciada ( )D- Outros

II- Questões Específicas

Qual série do curso de graduação em Enfermagem você está cursando no momento?

( ) A- $1^{a}$ série. Ano:

( ) B- $2^{a}$ série. Ano:

( ) C- $3^{a}$ série. Ano:

( ) D- $4^{a}$ série. Ano:

Você já assistiu algum tipo de parto? Qual?

( ) A-Cesariana

( ) B-Parto Normal

( ) C-Nunca assisti

Forma que the possibilitou assistir o parto:

(Caso você nunca tenha assistido pule esta questão).

( ) A-Durante disciplina escolar.

( ) B- Vídeo na internet.

PARTO NORMAL

( ) C- Outros
( ) A-Estágio durante a graduação.
( ) B- Vídeo na internet
PARTO CESÁRIA
( ) C-Outros

Você saberia informar qual via de parto ocorreu no momento de seu nascimento?
( ) A- Normal
( ) B- Cesariana
( ) C- Outros

Qual via de parto você escolheria?

( ) A- Normal

( ) B-Cesariana

Justifique sua resposta:

Você tem filho (s)? Se sim, especificar a quantidade.

A-( ) Sim, Quantidade:

B-( ) Não

Qual a via de parto ocorreu no momento do nascimento de seus filhos?

Resposta especifica de acordo com o número de partos.

$1^{\circ}$ filho (a): $\quad$ A-( ) Cesariana

B-( ) Parto Normal 
$2^{\circ}$ filho(a):

A-( ) Cesariana

B-( ) Parto Normal

Outros:

A-( ) Cesariana

A-( ) Cesariana

B-( ) Parto Normal

B-( ) Parto Normal

( ) Não tenho filhos.

A via de parto escolhida foi uma decisão sua?

Se você escolheu a opção NÃO TENHO FILHOS acima, pule esta questão.

( ) A- Foi uma decisão exclusivamente minha

( ) B- Foi uma decisão exclusivamente do obstétra

( ) C- Foi uma decisão minha e de meu obstetra.
Justifique o motivo da escolha do tipo de parto:

Qual seu conhecimento quanto ao papel do enfermeiro(a) obstetra?

Você gostaria que seu parto fosse realizado por este tipo de profissional? Justifique.

A-( ) Sim

B-( ) Não

Justificativa:

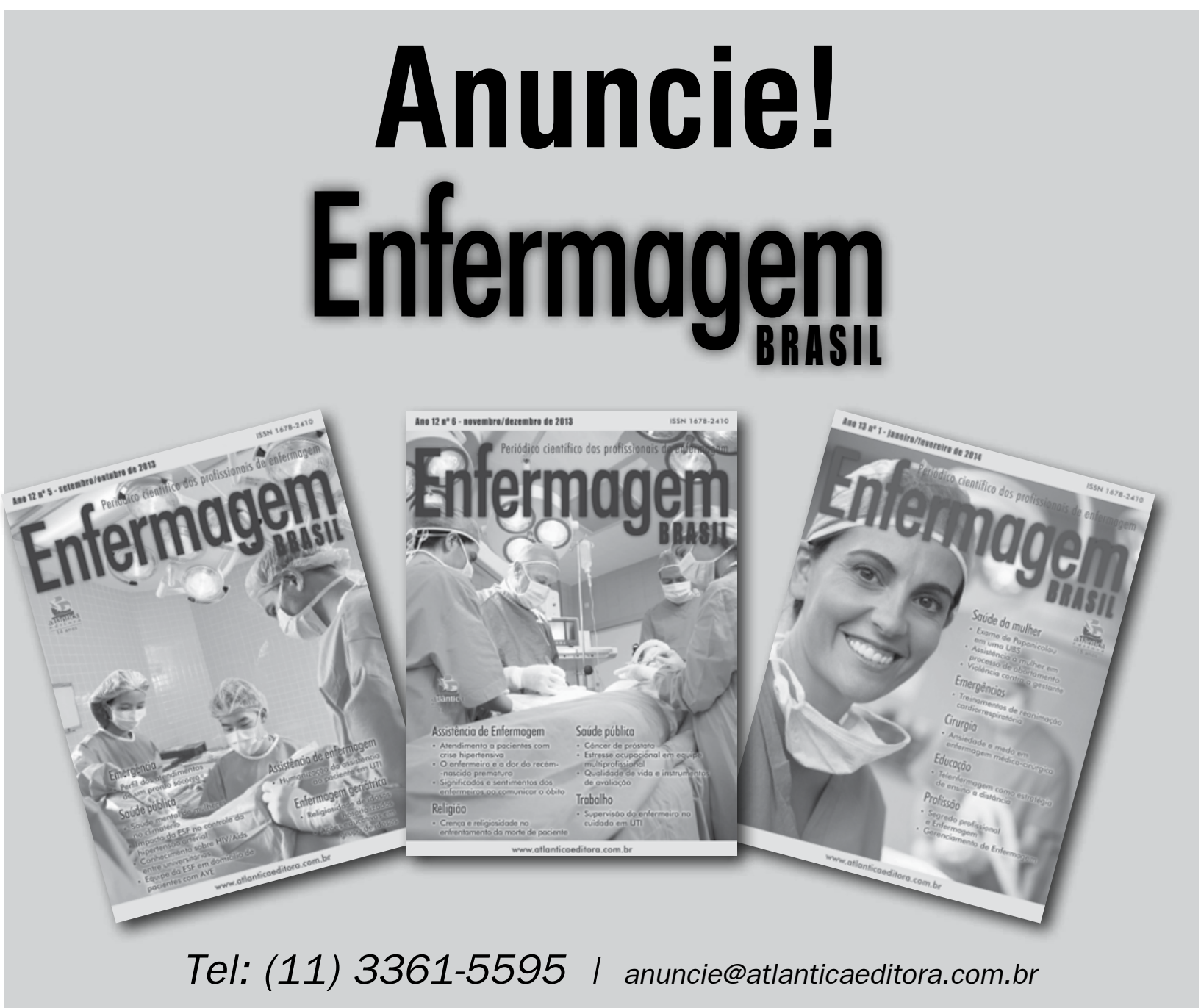

